\title{
ALTERAÇÕES HEMATOLÓGICAS NA LEPTOSPIROSE ${ }^{(1)}$
}

\section{A. C. NICODEMO (2), N. MEDEIROS (3) G. DEL NEGRO (4) \& V. AMATO NETO (5).}

\section{RESUMO}

Foram estudadas as alteraçōes hematológicas de 30 pacientes com diagnóstico de leptospirose internados na Clínica de Doenças Infecciosas e Parasitárias do Hospital das Clinicas da Faculdade de Medicina da Universidade de Sảo Paulo.

Verificou-se que mais da metade dos pacientes $16(53,3 \%)$ apresentavam contagem normal de leucócitos no momento da internaçāo; $12(40 \%)$ apresentavam leucó citos e $2(6,6 \%)$ leucopenia. Vinte e cinco doentes $(83,3 \%)$ apresentavam desvio à esquerda e $29(96,6 \%)$ neutrofilia relativa. Vinte e quatro pacientes tiveram o diagnós tico de anemia.

A plaquetopenia ocorreu em $26(86,6 \%)$ dos doentes.

Como alteraçōes mais características no mielograma, ressaltamos a alteraçāo da relaçāo G/E (série Granulocítica/série Eritroblástica), às custas de hipercelula ridade relativa e/ou absoluta da série granulocitica, e/ou hipocelularidade relativa e/ou absoluta da série vermelha; a eritropoese predominantemente microeritroblás tica em vários doentes; o encontro de plasmocitose discreta ou moderada e, na série intersticial, o aumento da atividade macrofágica.

A análise estatística da série megacariocitária do mielograma dos 30 pacientes revelou näo existir relação de dependência significativa entre a celularidade da série megacariocitária e o número de plaquetas no sangue periférico; porém, verificou-se existência da relaçāo de dependência entre a plaquetogênese medular e a contagem de plaquetas no sangue periférico. A possibilidade de haver inibiçāo da plaquetogênese medular por algum produto bacteriano náo pode ser totalmente afastada, porém acreditamos que o exame estático da medula óssea não pode dar-nos uma idéia precisa do mecanismo dinàmico da formação de plaquetas na presença de "consumo periférico".

UNITERMOS: Leptospirose; Alteraçōes laboratoriais.

(1) Trabalho realizado no Departamento de Doencas Infecciosas e Parasitárias da Faculdade de Medicina da Universidade de São Paulo (FMUSP). Sào Paulo, SP, Brasil.

(2) Professor Assistente do Departamento de Doenças Infecciosas e Parasitárias da FMUSP. Sāo Paulo. SP. Brasil

(3) Medico da Seçāo de Hematologia. Citologia e Genética Clínica do Laboratório Central do Hospital das Clínicas da FMUSP. Sào Paulo, SP. Brasil.

(4) Ex-Professor Adjunto - Departamento de Doenças Infecciosas e Parasitárias da FMUSP. Sào Paulo, SP. Brasil.

(5) Professor Titular do Departamento de Doenças Infecciosas e Parasitárias da FMUSP. São PauJo, SP. Brasil.

Endereço para correspondência: Dr. Antonio Carlos Nicodemo. R. Cristiano Viana, 191 - Apto. 52 . CEP 05411 Sảo Paulo. SP. Brasil. 
NICODEMO. A. C.: MEDEIROS. N.: DEL NEGRO. G. \& AMATO NETO. V. - Alteraçōes hematológicas na leptospirose Rev. Inst. Med. trop. São Paulo, 31 (2): 71 79. 1989

\section{INTRODUÇĀO}

A leptospirose é uma doença infecciosa aguda causada por espiroquetas patogénicas do gênero Leptospira podendo acometer o homem e animais domésticos e selvagens.

A leptospirose pode determinar no homem manifestaçóes clínicas variadas, desde as infec çōes inaparentes até a forma icterohemorrá gica, também conhecida como "Doença de weil".

O diagnóstico, mesmo nas formas graves, às vezes é dificil, principalmente na fase inicial e a reação de soroaglutinação microscópica, comumente se positiva somente a partir da segun da semana da doença, daí a importância dos ou tros exames laboratoriais no auxilio ao diagnóstico mais precoce.

O objetivo deste trabalho foi o de mostrar as alteraçōes hematológicas encontradas na for ma grave da leptospirose, fornecendo assim, sub sídios para o diagnóstico e o melhor conheci mento desses aspectos laboratoriais que ocorrem na doença.

\section{CASUISTICA E MÉTODOS}

\section{A) População Estudada}

Neste estudo, que corresponde a parte de nossa Dissertaçāo de Mestrado ${ }^{9}$, foram estuda dos trinta pacientes com diagnóstico de leptospirose internados na Clínica de Doenças Infec ciosas e Parasitárias do Hospital das Clínicas da Faculdade de Medicina da Universidade de São Paulo sendo que, a seleção dos pacientes fez-se independentemente do valor da contagem das plaquetas no momento da internaçào.

Foram analisadas algumas características dessa populaçāo tais como idade, sexo e cor.

\section{B) Métodos}

\section{B.1 - Sorologia para leptospirose}

Foi utilizada a reaçào de soroaglutinaçāo microscópica, realizada no Instituto Adolfo Lutz de São Paulo.

Como critérios para diagnóstico foram utili zados os adotados pelo United States Depart ment of Health, Education and Welfare ${ }^{6}$.

\section{B. 2 - Hemograma}

As contagens globais de leucócitos e eritró citos, as dosagens de hemoglobina, as determinaçōes do hematócrito foram realizadas em contador automático Coulter, modelo "S". As contagens diferenciais leucocitárias foram realizadas em esfregaços de sangue após a prévia coloração pelo método de Leishman.

Valores normais para contador automático Coulter, modêlo " $\mathrm{S}$ ":

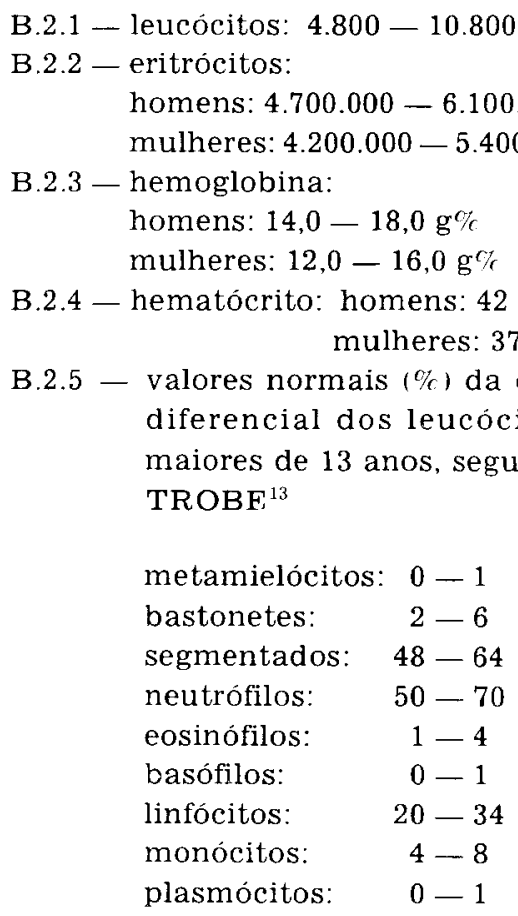

Para a caracterização de anemia utilizaram se os valores de hematócrito menores que $42 \%$ para os homens e menores que $37 \%$ para as mulheres, e os valores de hemoglobina menores que $14 \mathrm{~g} \%$ para os homens e menores que $12,0 \mathrm{~g} \%$ para as mulheres, segundo WINTROBE ${ }^{13 .}{ }^{14}$ além dos valores considerados para o contador auto mático Coulter, modêlo " $\mathrm{S}$ ".

\section{B.3 - Mielograma}

Os territórios ósseos puncionados eram manú brios esternais em seis casos (20\%) e espinhas ântero-superiores das cristas ilíacas direita ou esquerda, em 24 casos $(80 \%)$. 
NICODEMO, A. C.: MEDEIROS, N.; DEL NEGRO, G. \& AMATO NETO, V. - Alteraçoes hematologicas na leptospirose. Rev. Inst. Med. trop. Sâo Paulo, 31 (2): 71-79, 1989.

As amostras medulares, imediatamente após as coletas, foram estendidas em lâminas ${ }^{5}$ Esses esfregaços de medula óssea depois de secos foram submetidos à coloração pancrômica (Leishman).

Os relatórios das medulas ósseas foram fei tos de forma descritiva, enfatizando os seguintes itens: celularidade, relaçāo $\mathrm{G} / \mathrm{E}$, setores granulocítico, eritrocítico, linfomonoplasmocitário, megacariocitário e intersticial.

\section{B.4 - Contagem das plaquetas}

Fez-se contagem direta pelo método de
BRECHER \& CRONKITE ${ }^{3}$. Valor normal: 150.000 a $450.000 / \mathrm{mm}^{3}$.

\section{RESULTADOS}

\section{A. CARACTERISTICAS EPIDEMIOLÓGICAS DA POPULAḈ⿴囗 ESTUdADA.}

$$
\text { A.1 - Idade }
$$

A idade variou entre os 13 e os 70 anos e a distribuiçáo dos casos é mostrada na tabela 1 .

TABELA 1

\begin{tabular}{|c|c|c|c|c|c|c|}
\hline Caso & Sexo & Idade & $\begin{array}{c}\mathrm{N} \text {. de leucócitos } \\
\left(/ \mathrm{mm}^{3}\right)\end{array}$ & \% de neutrófilos & $\begin{array}{l}\text { N: absoluto de } \\
\text { neutrófilos }\end{array}$ & $\begin{array}{l}\mathrm{N} \text {. de eritrócitos } \\
\left(\times 10^{5} / \mathrm{mm}^{3}\right)\end{array}$ \\
\hline 1 & $\mathbf{M}$ & 27 & 11.600 & 81 & 9.396 & 3.59 \\
\hline 2 & $\mathbf{M}$ & 22 & 8.100 & 81 & 6.561 & 4.10 \\
\hline 3 & M & 60 & 22.500 & 93 & 20.925 & 3.20 \\
\hline 4 & M & 19 & 18.400 & 86 & 15.824 & 3.80 \\
\hline 5 & $\mathrm{M}$ & 22 & 8.000 & 87 & 6.960 & 4.33 \\
\hline 6 & M & 36 & 6.500 & 87 & 5.655 & 3.70 \\
\hline 7 & M & 13 & 14.700 & 89 & 13.083 & 3.90 \\
\hline 8 & $\mathbf{M}^{x}$ & 49 & 6.200 & 79 & 4.898 & NR \\
\hline 9 & M & 40 & 6.000 & 89 & 5.340 & NR \\
\hline 10 & M & 18 & 9.700 & 85 & 8.245 & NR \\
\hline 11 & $\mathrm{~F}$ & 70 & 16.400 & 95 & 15.580 & NR \\
\hline 12 & $\mathrm{M}$ & 41 & 7.000 & 90 & 6.300 & 4.50 \\
\hline 13 & M & 35 & 10.700 & 81 & 8.667 & 2.80 \\
\hline 14 & M & 48 & 6.900 & 81 & 5.589 & 4.40 \\
\hline 15 & M & 15 & 9.000 & 79 & 7.110 & 4.43 \\
\hline 16 & $\mathbf{M}$ & 29 & 16.000 & 91 & 14.560 & 5.00 \\
\hline 17 & $\mathrm{M}$ & 42 & 24.300 & 83 & 20.169 & 2.29 \\
\hline 18 & M & 62 & 18.400 & 97 & 17.848 & 2.91 \\
\hline 19 & $\mathbf{M}$ & 14 & 4.500 & 76 & 3.420 & 4.22 \\
\hline 20 & M & 33 & 7.800 & 92 & 7.176 & 4.13 \\
\hline 21 & M & 58 & 15.000 & 84 & 12.600 & 4.40 \\
\hline 22 & M & 22 & 4.600 & 73 & 3.358 & 3.70 \\
\hline 23 & M & 27 & 10.700 & 79 & 8.374 & NR \\
\hline 24 & $\mathbf{M}$ & 35 & 10.100 & 90 & 9.090 & 3.71 \\
\hline 25 & $\mathrm{~F}$ & 39 & 16.500 & 86 & 14.190 & 2.69 \\
\hline 26 & M & 50 & 8.600 & 83 & 7.138 & 3.20 \\
\hline 27 & M & 28 & 27.100 & 89 & 24.119 & 3.07 \\
\hline 28 & M & 48 & 14.000 & 81 & 11.340 & 4.48 \\
\hline 29 & M & 46 & 9.200 & 62 & 5.704 & 4.17 \\
\hline 30 & $M$ & 30 & 8.800 & 90 & 7.920 & 4.16 \\
\hline
\end{tabular}

* NR - Não realizado 
NICODEMO, A. C.; MEDEIROS, N.; DEL NEGRO. G.\& AMATO NETO. V. - Alteraçóes hematológicas na leptospirose. Rev. Inst. Med. trop. Sáo Paulo, 31 (2): 71-79, 1989.

\section{A.2 - Sexo}

Vinte e oito pacientes $(93.3 \%)$ eram do sexo masculino e dois $(6,6 \%)$ do sexo feminino.

$$
\text { A.3 - Cor }
$$

Dezoito doentes $(60 \%)$ eram brancos, sete $(23,3 \%)$ mulatos, quatro $(13,3 \%)$ negros e um $(3,3 \%)$ amarelo.

\section{RESULTADOS}

\section{B. HEMOGRAMA}

B.1-Contagem dos leucócitos.

Dezesseis pacientes $(53,3 \%)$ apresentavam contagem normal de leucócitos no momento da internação; $12(40 \%)$ apresentavam leucocitose e dois $(6,6 \%)$ leucopenia. O número mínimo absoluto foi de 4.500 leucócitos por $\mathrm{mm}^{3}$. Os números extremos nos pacientes que apresentaram leu cocitose foram $11.600 / \mathrm{mm}^{3}$ e $27.100 / \mathrm{mm}^{3}$.

B.2 - Desvio à esquerda.

Vinte e cinco doentes $(83,3 \%)$ apresentavam desvio à esquerda no hemograma inicial

B.3 - Presença de neutrofilia.

Vinte e nove pacientes $(96,6 \%)$ apresenta vam neutrofilia relativa e dezessete $(56,6 \%)$ apre sentavam neutrofilia absoluta no hemograma inicial.

B.4-Contagem dos eritrócitos.

A contagem dos eritrócitos realizada em 25 pacientes no hemograma inicial mostrou reduçāo no número de hemácias em 24 (96\%).

B.5 - Valores de hemoglobina no hemo grama inicial.

Dos 30 pacientes estudados, $28(93,3 \%)$ apresentaram valores diminuidos de hemoglobina. Os valores extremos foram 6,9 e $14,6 \mathrm{~g} \%$ (Tabela 2).

TABELA 2

Distribuiçāo dos pacientes segundo os valores e hemoglobina no hemograma inicial.

\begin{tabular}{cccccc}
\hline Valores (g\%) & $6,9-8,5$ & $8,6-10,3$ & $10,4-12,1$ & $12,2-13,9$ & 14 \\
\hline Número de pacientes & 3 & 5 & 11 & 9 & 2 \\
\hline
\end{tabular}

B.6 - Valores do hematócrito no hemograma inicial.

Vinte e quatro de 25 doentes nos quais foi feita a determinaçāo do hematócrito tinham va- lores abaixo do normal. Em um paciente to n? 16), o hematócrito estava normal.

Os valores extremos foram 22,1 e $43 \%$ ( $\mathrm{Ta}$ bela 3 ).

TABELA 3

Distribuição dos pacientes segundo os valores de hematócrito no hemograma inicial.

\begin{tabular}{cccccc}
\hline Valores $(\%)$ & $22,1-27,0$ & $27,1-32,0$ & $32,1-37,0$ & $37,1-42,0$ & $42,1-47,0$ \\
\hline Número de pacientes & 3 & 4 & 8 & 9 & 1 \\
\hline
\end{tabular}

B.7 - Anemia

Dois pacientes ( $\mathrm{n}$ ? 11 e 25 ) eram do sexo feminino e o restante do sexo masculino.
Baseados nas determinações do hematócrito e da hemoglobina, os seguintes pacientes foram caracterizados como portadores de anemia: 
NICODEMO, A. C.; MEDEIROS, N.; DEL NEGRO, G. \& AMATO NETO, V. - Alteraçóes hematológicas na leptospirose. Rev. Inst. Med. trop. São Paulo, 31 (2): 71-79, 1989

$1,2,3,4,5,6,7,12,13,15,17,18,19,20,21,22$, $23,24,25,26,27,28,29$ e 30 .

Os doentes n: 8, 9, 10,11 e 14 apresentavam valores de hemoglobina de $9,6,14,0,12,2,9,3 \mathrm{e}$ $13,7 \mathrm{~g} \%$ respectivamente, mas não tiveram determinação do hematócrito em razāo de proble mas técnicos com o contador, fato que os deixou portanto, excluidos dessa análise. O paciente $n^{\circ}$ 16 apresentava valores normais de hematócrito e hemoglobina.

\section{MIELOGRAMA}

A medula mostrou-se normocelular em 19 $(63,3 \%)$, hipocelular em $6(20 \%)$ e hipercelular em $5(16,6 \%)$.

A série granulocítica apresentou hipercelularidade relativa em 9 casos $(30 \%)$ e relativa e absoluta em 9 casos (30\%). Em oito doentes $(26,6 \%)$, a série granulocitica era normocelular. Em três pacientes (10\%) havia hipocelularidade absoluta e em um $(3,3 \%)$, hipocelularidade relativa.

O processo maturativo do setor neutrófilo estava conservado em 15 casos ( $50 \%$ ); em 11 pacientes $(36,6 \%)$ havia anaplasia somente em promielócitos neutrófilos e em três (10\%) havia anaplasia em promielócitos e mielócitos neutrófilos. Um doente tinha escalonamento maturativo do setor neutrófilo com aumento da taxa de formas segmentadas.

Os eosinófilos achavam-se em número normal em 29 doentes $(96,6 \%)$ e aumentado em um $(3,3 \%)$. O número de basófilos era normal nos 30 pacientes $(100 \%)$.

A série vermelha apresentava hipocelularidade relativa em $12(40 \%)$, absoluta e relativa em seis $(20 \%)$ e absoluta em três $(10 \%)$ doentes. Oito pacientes $(26,6 \%)$ tinham a série vermelha normocelular. Em um doente $(3,3 \%)$ havia hipercelularidade relativa e absoluta.

A maturação do setor eritroblástico apresentava-se normal em 27 pacientes $(90 \%)$. Em dois doentes $(6,6 \%)$ não foi possivel avaliar o escalonamento maturativo; em um havia aumento do número de eritroblastos ortocromatófilos (EOC)
A eritropoese era predominantemente $\mathrm{mi}$ croeritroblástica em $12(40 \%)$; cinco $(16,6 \%)$ apre sentavam raros microeritroblastos: cinco $(16,6 \%)$ vários microeritroblastos; um $(3,3 \%) \mathrm{ra}$ ros micro e macronormoblastos; um $(3,3 \%)$ vários micro e macronormoblastos; um $(3,3 \%)$ pre dominantemente normoblástico; um (3,3\%) nor moblástica com componente microeritroblástico; um $(3,3 \%)$ predominantemente macroeritroblástico (com poucos elementos do tipo normo e raros megaloblastóides).

A eritropoese apresentava-se polimorfa em um $(3,3 \%)$, com componentes micro e macroeri troblásticos. Em um $(3,3 \%)$ a eritropoese era normoeritroblástica e em um $(3,3 \%)$ a eritropoese ficou com avaliação prejudicada.

A relação $\mathrm{G} / \mathrm{E}$ (série granulocítica/série eritroblástica) foi em média de 4,62/1. Em um doen te $(3,3 \%)$ a avaliaçāo da relação ficou prejudicada.

A série linfomonoplasmocitária revelou-se normal em 19 casos $(63,3 \%)$. Em 11 pacientes $(36,6 \%)$ havia plasmocitose (discreta ou moderada).

Na série intersticial houve aumento da atividade macrofágica em 17 pacientes $(56,6 \%)$; em 13 doentes $(43,3 \%)$ a série intersticial estava nor mal.

A série megacariocitária é relatada e analisada em separado.

$$
\begin{aligned}
& \text { C.1 - Mielograma - Série Megacariocitá- } \\
& \text { ria (Tabela 4). }
\end{aligned}
$$

A série megacariocitária era normocelular em 24 pacientes, dos quais 13 tinham hipopla quetogênese. Todos esses pacientes apresentavam plaquetopenia no sangue periférico. Em 11 desses 24 , a medula era normoplaquetogênica, porém sete desses casos apresentavam plaque topenia no sangue periférico.

Em cinco doentes a série megacariocitária era hipocelular sendo hipoplaquetogênica em três cos três apresentavam plaquetopenia no sangue periférico) e normoplaquetogènica em dois; estes dois também eram plaquetopênicos. 
NICODEMO, A. C.: MEDEIROS, N.: DEL NEGRO. G. \& AMATO NETO, V. - Alteraçóes hematológicas na leptospirose Rev. Inst. Med. trop. São Paulo, 31 (2): 71 79, 1989

Em um paciente, a série era hipercelular. porém hipoplaquetogenica e o paciente apresen. tava plaquetopenia no sangue periférico.

A análise estatistica desse material (série megacariocitária do mielograma inicial dos 30 pacientes) revelou não existir relação de dependência significativa entre a celularidade da série megacariocitária e o número de plaquetas no sangue periférico: $P\left(\mathrm{X}^{2} \mathrm{c}>1,244\right)=0,5369$.

Verificou-se, no entanto, manutenção da re lação de dependência entre a plaquetogênese medular e o número de plaquetas no sangue periférico: $\mathrm{p}=0,0261$.

TABELA 4

Mielograma: Série Megacariocitárı

(30 pacientes)

\begin{tabular}{|c|c|c|c|c|c|}
\hline Celular & dade & Plaquetog & ênese & Plaquetas no sangu & e periférico \\
\hline & & Hipoplaquetogenica & $-(13)$ & Plaquetopenia & $-(13)$ \\
\hline Normocelular & $-(24)$ & Normoplaquetogènica & $-(11)$ & $\begin{array}{l}\text { Plaquetopenia } \\
\text { Plaquetas normais }\end{array}$ & $\begin{array}{l}-\quad 7) \\
-\quad(4)\end{array}$ \\
\hline Hipocelular & -151 & Hipoplaquetogênica & $-(3)$ & Plaquetopenia & $-(3)$ \\
\hline & & Normoplaquetogénica & $-(2)$ & Plaquetopenia & $-\quad(2)$ \\
\hline Hipercelular & - (1) $_{1}$ & Hipoplaquetogènica & $-(1)$ & Plaquetopenia & $-(1)$ \\
\hline
\end{tabular}

$1,=$ Número de casos.

TABELA 5

\begin{tabular}{|c|c|c|c|}
\hline Caso & $\begin{array}{l}\mathrm{N}: \text { de plaquetas } \\
\qquad\left(\mathrm{mm}^{3}\right)\end{array}$ & $\begin{array}{c}\text { Tempo de } \\
\text { normalizacao de } \\
\text { plaquetas rdias }\end{array}$ & $\begin{array}{l}\text { Presenca de } \\
\text { sangramento }\end{array}$ \\
\hline 1 & 50.000 & 7 & SIM \\
\hline 2 & 40.000 & 4 & SIM \\
\hline 3 & 10.000 & 6 & SIM \\
\hline 4 & 45.000 & 4 & SIM \\
\hline 5 & 15.000 & 6 & SIM \\
\hline 6 & 155.000 & - & NAO \\
\hline 7 & 165.000 & $\ldots$ & NÁO \\
\hline 8 & 30.000 & 7 & $\mathrm{NABO}$ \\
\hline 9 & 80.000 & 6 & NAO \\
\hline 10 & 125.000 & 2 & $\mathrm{NAO}$ \\
\hline 11 & 35.000 & 4 & SIM \\
\hline 12 & 9.000 & 7 & NAO \\
\hline 13 & 95.000 & 13 & SIM \\
\hline 14 & 65.000 & 7 & NAO \\
\hline 15 & 20.000 & 5 & NAO \\
\hline 16 & 240.000 & $\ldots$ & NẢO \\
\hline 17 & 40.000 & 7 & SIM \\
\hline 18 & 31.000 & 2 & SIM \\
\hline 19 & 60.000 & 1 & SIM \\
\hline 20 & 40.000 & 5 & NAO \\
\hline 21 & 280.000 & -- & NAOO \\
\hline 22 & 70.000 & 8 & NÁO \\
\hline 23 & 100.000 & 3 & NAOO \\
\hline 24 & 16.000 & 6 & $\mathrm{NABO}$ \\
\hline 25 & 25.000 & 6 & SIM \\
\hline 26 & 60.000 & 6 & SIM \\
\hline 27 & 63.000 & 23 & SIM \\
\hline 28 & 39.000 & 7 & SIM \\
\hline 29 & 80.000 & 3 & SIM \\
\hline 30 & 5.000 & 12 & SIM \\
\hline
\end{tabular}

\section{CONTAGEM DE PLAQUETAS}

D.1 - Plaquetopenia (ver Tabela 5)

A plaquetopenia ocorreu em $26(86,6 \%)$ dos 30 doentes

O número inicial de plaquetas variou de $5.000-125.000 / \mathrm{mm}^{3}$ (média $48.000 \pm$ $\left.30.649 / \mathrm{mm}^{3}\right)$. Em quatro pacientes $(13,3 \%)$, não ocorreu plaquetopenia.

D.2 - Tempo para normalizaçào do número de plaquetas a partir do exame inicial.

O tempo para normalização da contagem de plaquetas variou de 2 a 23 dias.

Dezenove doentes $(86,3 \%)$ de 22 tiveram a contagem de plaquetas normalizadas até o sétimo dia da in ternação, conforme mostra a Tabela 5 .

OBS: Quatro pacientes permaneceram plaquetopénicos até o momento do óbito, respectivamente $1,2,6$, e 13 dias, caso dos pacientes n: $19,18,3$ e 13 . 
D.3 - Plaquetopenia e sangramento ( $\mathrm{Ta}^{--} 115$ pacientes com leptospirose internados no bela 5 ).

Dos 26 pacientes portadores de plaquetopenia no momento da internação, $16(61,5 \%)$ apresentaram sangramento.

O número de plaquetas nesses 16 pacientes variou de 5.000 a $95.000 / \mathrm{mm}^{3}$ (média $43.312 \pm$ $24.475 / \mathrm{mm}^{3}$ ).

O número de plaquetas nos 10 pacientes plaquetopênicos que nāo apresentaram sangramento variou de 9.000 a $125.000 / \mathrm{mm}^{3}$ (média $55.500 \pm 38.851 / \mathrm{mm}^{3}$ )

Os pacientes n: 6, 7, 16 e 21 apresentavam contagem normal de plaquetas no momento da internaçào e não apresentaram sangramento. $\mathbf{E}$ importante salientar que todos os 16 doentes que apresentaram sangramento eram plaquetopéni cos.

O tempo médio para a normalização da con tagem de plaquetas nos pacientes plaquetopé nicos que apresentaram sangramento foi de sete dias e nos que não apresentaram sangramento foi de cinco dias.

Não existe diferença significativa entre as médias dos tempos para a normalização da contagem das plaquetas nos pacientes que apresentaram e os que não apresentaram sangramento: $t_{16}=1,057<t_{16} ; 0,05=1,746$.

A comparação estatística entre os grupos com sangramento e os sem sangramento nâo mostrou diferença significativa para a contagem das plaquetas:

$$
\mathrm{P}(\mathrm{t}>0,888)=0,1917 .
$$

\section{DISCUSSĀO}

Pouco mais da metade de nossos pacientes $(53,3 \%)$ apresentava contagem normal de leucócitos no momento da internaçāo (Tabela 1). A maioria apresentava neutrofilia relativa $(96,6 \%$ ) e desvio à esquerda $(83,3 \%)$ no leucograma inicial.

Nossos resultados com relação à série bran ca são semelhantes aos descritos em nosso meio por TOMAZINI e col. (1983) $)^{11}$, os quais chegaram a uma con tagem normal de leucócitos em $48,6 \%$, leucocitose em $38,5 \%$ e leucopenia em $12,8 \%$ de Hospital "Emilio Ribas". Esses autores encontraram também desvio à esquerda e neutrofilia na maioria dos doentes estudados.

Independentemente da contagem dos leucócitos, a neutrofilia relativa parece ser a regra nes sa forma da doença.

Prosseguindo na análise das alteraçoes hematológicas constatamos anemia em 24 pacien. tes.

É importante salientar que devido à escas sez em nosso meio de dados relativos aos valores normais de hematócrito e hemoglobina, optamos por utilizar, na caracterizaçāo da anemia, os valores abaixo dos minimos normais segundo WINTROBE ${ }^{13}$, e para o contador automático Coulter, para essas determinaçōes.

Obtivemos resultados semelhantes aos descritos por URICH e col. (1987) $)^{12}$ que encontraram $90,3 \%$ d $\epsilon$ anemia em 31 pacientes com leptos pirose internados no Hospital "Emilio Ribas" no geriodo de fevereiro a junho de 1986.

Como alteraçōes mais características no mielograma, aspecto pouco estudado na leptos pirose $^{1.8 .12}$, ressaltamos a alteração da relaçāo G/E (série granulocítica/série eritroblástica), às custas de hipercelularidade relativa e/ou abso. luta da série granulocítica, e/ou hipocelularidade relativa e/ou absoluta da série vermelha; a eritropoese predominantemente microeritro blástica em vários doentes; o encontro de plas mocitose discreta ou moderada e, na série inters ticial, o aumento da atividade macrofágica.

Na série megacariocitária, constatou-se que todos os 17 doentes, cujas séries megacarioci. tárias eram hipoplaquetogénicas, apresentavam plaquetopenia no sangue periférico, porém nove de treze pacientes que apresentavam série megacariocitária normoplaquetogénica também ti nham a contagem de plaquetas diminuída no sangue periférico (Tabela 4 ).

A análise estatística desse material (série megacariocitária dos 30 pacientes) revelou não existir relaçāo de dependencia significativa en. tre a celularidade da série megacariocitária e o número de plaquetas no sangue periférico:

$\mathrm{P}\left(\mathrm{X}^{2}{ }_{\mathrm{c}}>1,244\right)=0,5369$ 
NICODEMO, A. C.; MEDEIROS, N.; DEL NEGRO. G. \& AMATO NFTO. V. - Alteraçoes hematológicas na leptospirose Rev. Inst. Med. trop. São Paulo, 31 (2): $71-79.1989$.

Verificou-se, no entanto, que apesar da exis tência de pacientes com série megacariocitária normoplaquetogènica e plaquetopenia no san gue periférico, houve relaçāo de dependência en tre a plaquetogênese medular e a contagem de plaquetas no sangue periférico: $\mathrm{P}=0,0261$.

A possibilidade de haver inibição da plaque togênese medular por algum produto bacteriano (toxina) não pode ser totalmente afastada, po rém acreditamos que o exame estático da medu la óssea não nos pode dar uma idéia precisa do mecanismo dinâmico da formaçāo de plaquetas quando da presença de consumo periférico.

Verificamos que $26(86,6 \%)$ dos pacientes apresentaram plaquetopenia (Tabela 5).

A literatura mostra variaçôes regionais im portantes na prevalência de plaquetopenia na Leptospirose $\mathrm{L}^{2,4,5,7,10,11}$, o que, a nosso ver, está relacionado com o amplo espectro de apresen taçōes da doença que parece depender não somente do hospedeiro mas, principalmente, do sorotipo e da virulência da cepa infectante.

A maioria dos nossos doentes apresentavam a forma grave da doença e o percentual de plaquetopênicos por nós encontrado é semelhante ao descrito por TOMAZINI e col. $(1983)^{11}$, os quais encontraram $79,2 \%$ de plaquetopenia em 101 casos de leptospirose internados no Hospital "Emílio Ribas" no período de janeiro de 1981 e janeiro a dezembro de 1982 .

Podemos dizer que a plaquetopenia é fre qüentemente observada nas formas graves de leptospirose ocorridas em nosso meio.

Em 19 de 22 pacientes $(86,3 \%)$ a normaliza ção da contagem de plaquetas ocorreu até o sétimo dia da internação (Tabela 5) mostrando que, de modo geral, o retorno para os valores normais costuma ocorrer precocemente durante a internaçäo.

Dos 26 pacientes portadores de plaquetope nia no momento da internação, $16(61,5 \%)$ apresentaram sangramento (Tabela 5). Esse grupo correspondeu ao total dos pacientes do nosso trabalho que apresentaram sangramento.

A comparação estatística entre os grupos de plaquetopênicos que apresentaram e os que não apresentaram sangramento mostrou nảo haver diferença significativa para a contagem de pla quetas. Também não ocorreu diferença signifi cativa entre as médias dos tempos de norma lizaçāo da contagem de plaquetas nos pacientes que apresentaram e os que nào apresentaram sangramento.

Podemos dizer que, embora a plaquetopenia possa representar um fator contribuinte para a sindrome hemorrágica que ocorre na doença, sua participaçāo no quadro hemorrágico se faz de maneira secundária, sendo a lesão capilar provavelmente o fator primário e o mais impor tante no desencadeamento dos fenomenos he morrágicos que ocorrem na leptospirose.

\section{SUMMARY}

\section{Hematologic disorders in leptospirosis.}

30 cases of leptospirosis admitted to the Cli nica de Doenças Infecciosas e Parasitárias do Hospital das Clínicas da Faculdade de Medicina da Universidade de Sáo Paulo were studied for blood count alterations. 16 patients $(53,3 \%)$ had a normal white blood cell count at the moment of admission. 12 patients $(40 \%)$ presented a high white blood cell count and $2(6,6 \%)$ had a low count. 29 patients $(96,6 \%)$ had a high proportion of neutrophils and 25 patients $(83,3 \%)$ presented a high number of immature forms.

24 patients had anemia.

Thrombocytopenia was present in $26 \mathrm{pa}$ tients $(86.6 \%)$.

The most characteristic changes found in bone marrow aspirate were the alterations of $M$ : $E$ ratio (myeloid - erythroid ratio) due to rela tive and/or absolute hyperplasia of the myeloid series, and/or relative and/or absolute hypopla sia of the erythroid series; erythropoiesis was predominantly microerythroblastic in many patients; mild to moderate plasmocytosis was found; and, regarding the intersticial series, increased macrophagic activity was noted.

There was no direct correlation between the number of megakaryocytes and the blood plate let count, but there was a direct correlation bet 
NICODEMO, A. C.; MEDEIROS, N.; DEL NEGRO, G. \& AMATO NETO, V. - Alteraçōes hematológicas na leptospirose. Rev. Inst. Med. trop. Sāo Paulo, 31 (2): 71-79, 1989

ween the bone marrow platelet production and blood platelet count.

We believe that it is very difficult to have a good idea of the dynamic mechanisms that lead to medular platelet production in the presence of platelet consumption, through a ran dom test of the bone marrow

\section{REFERÊNCIAS BIBLIOGRÁFICAS}

1. AREAN. V. M. - The pathogenic anatomy and pathoge nesis of fatal human leptospirosis (Weil's Disease). Amer. J. Path., 40: 393-423, 1962.

2. BERMAN, S. J.; TSAI, C.; HOLMES, K.; FRESH, J. W \& WATTEN, R. H. - Sporadic anicteric leptospirosis in South Vietnam: a study in 150 patients. Ann. intern. Med., 79: $167-173,1973$

3. BRECHER, G. \& CRONKITE, E. P. - Morphology and enumeration of human blood platelets. J. appl. Physiol. 3: $365-377,1950$

4. EDWARDS, C. N ; NICHOLSON, G. D. \& EVERARD, C O. R. - Thrombocy topenia in leptospirosis. Amer. J. trop. Med. Hyg., 31: 827-829, 1982

5. EDWARDS, C. N.; NICHOLSON. G. D. HASSEL. T. A EVERARD, C. O.R. \& CALLENDER, J. - Thrombocy to penia in leptospirosis: the absence of evidence for dissemi nated intravascular coagulation. Amer. J. trop. Med. Hyg., 35: $352-354,1986$

6. FEIGIN, R. D. \& ANDERSON. D. C. - Human leptos pirosis. Crit. Rev. Clin. Lab. Sci., 5: 445, 1975
7. HEATH JR., C. W.; ALEXANDER, A D. \& GALTON, M. M. - Leptospirosis in the United States. New Engl. J. Med., $273: 915-922,1965$.

8. KHAN, J. B. - A case of Weil's disease requiring steroid therapy for thrombocytopenia and bleeding. Amer. J. trop. Med. Hyg., 31: 1213-1215, 1982

9. NICODEMO, A C. - Contribuiçāo ao estudo da plaquetopenia na leptospirose. São Paulo, 1987. (Dissertaçáo de Mestrado - Faculdade de Medicina da Universidade de Sāo Paulol.

10. RAOULT, D.; JEANDEL, P.; MAILlOUX, M. \& ROU GIER, Y. Thrombocy topenia and renal failure in leptos pirosis. Amer. J. trop. Med. Hyg., 32: 1464, 1983

11. TOMAZINI, M. F. M. F.: DIAMENT, D.: MIRANDA, A F. B.; SEGURO, A. C. \& LOMAR, A. V. - Leptospirose - Estudo retrospectivo de 115 casos internados no Hospital "Emilio Ribas". III. Aspectos hematológicos. In: CONGRESSO BRASILEIRO DE INFECTOLOGIA, 2, Sāo Paulo, 1983. Resumos. p. 54.

12. URICH, A. E.; LOMAR, A. V.; RUIZ, F. J.; SILVA, M R. R.; SUGANO, E. Y. \& KERBAUY, J. - Eritropoese na leptospirose. Rev. Soc. bras. Med. trop., 20 (supl): 139, 1987

13. WINTROBE, M. M. - Leucócitos. In: WINTROBE, M - Hematologia clínica. $3^{\text {a }}$ ed. Buenos Aires, Intermédica Editorial, 1969. p. 200-201

14. WiNTROBE, M. M. - In: WiNTROBE, M. M. - Clinical Hematology. $8^{\text {th }}$ ed. Philadelphia, Lea \& Fébiger, 1981. p. 1885.

Recebido para publicaçäo em 17/8/1988 